\title{
The health belief model and factors associated with adherence to treatment recommendations for positional plagiocephaly
}

\author{
Sandi Lam, MD, MBA, ${ }^{1}$ Thomas G. Luerssen, MD, ${ }^{1}$ Caroline Hadley, MD, ${ }^{1}$ Bradley Daniels, BS, ${ }^{1}$ \\ Ben A. Strickland, MD, ${ }^{1}$ Jim Brookshier, CPO, LPO, ${ }^{2}$ and I-Wen Pan, PhD' \\ 'Department of Neurosurgery/Division of Pediatric Neurosurgery, Texas Children's Hospital/Baylor College of Medicine; and \\ ${ }^{2}$ Hanger Orthotics, Houston, Texas
}

\begin{abstract}
OBJECTIVE This study aimed to examine factors associated with adherence to recommended treatment among pediatric patients with positional skull deformity by reviewing a single-institution experience (2007-2014) with the treatment of positional plagiocephaly.
\end{abstract}

METHODS A retrospective chart review was conducted. Risk factors, treatment for positional head shape deformity, and parent-reported adherence were recorded. Univariate and multivariate analyses were used to assess the impact of patient clinical and demographic characteristics on adherence.

RESULTS A total of 991 patients under age 12 months were evaluated for positional skull deformity at the Texas Children's Hospital Cranial Deformity Clinic between 2007 and 2014. According to an age- and risk factor-based treatment algorithm, patients were recommended for repositioning, physical therapy, or cranial orthosis therapy or crossover from repositioning/physical therapy into cranial orthosis therapy. The patients' average chronological age at presentation was 6.2 months; $69.3 \%$ were male. The majority were white (40.7\%) or Hispanic (32.6\%); $38.7 \%$ had commercial insurance and $37.9 \%$ had Medicaid. The most common initial recommended treatment was repositioning or physical therapy; $85.7 \%$ of patients were adherent to the initial recommended treatment.

Univariate analysis showed differences in adherence rates among subgroups. Children's families with Medicaid were less likely to be adherent to treatment recommendations (adherence rate, $80.2 \%$ ). Families with commercial insurance were more likely to be adherent to the recommended treatment $(89.6 \%)$. Multivariate logistic regression confirmed that factors associated with parent-reported adherence to recommended treatment included primary insurance payer, diagnosis (plagiocephaly vs brachycephaly), and the nature of the recommended treatment. Families were less likely to be adherent if they had Medicaid, a child with a diagnosis of brachycephaly, or were initially recommended for cranial orthosis therapy than families with commercial insurance, a child with a diagnosis of plagiocephaly, or an initial recommendation for repositioning or physical therapy.

Factors associated with treatment completion included corrected age, insurance, diagnosis, recommended treatment, and distance to provider from patient's residence. Patients with commercial insurance $(\mathrm{OR} 1.49,95 \% \mathrm{Cl} 1.10-2.02, p=$ $0.009)$, those diagnosed with both brachycephaly and plagiocephaly (OR 2.26, 95\% $\mathrm{Cl} 1.31-3.90, p=0.003)$, those recommended for treatment with cranial orthosis (OR $4.55,95 \% \mathrm{Cl}=3.24-6.38, p<0.001)$, and those living in proximity to the provider (OR 1.40,95\% $\mathrm{Cl} 1.00-1.96, p=0.047$ ) were more likely to complete treatment.

CONCLUSIONS Insurance type, degree of head shape deformity, and types of recommended treatment appear to affect rates of adherence to recommended treatments for positional skull deformation.

https://thejns.org/doi/abs/10.3171/2016.9.PEDS16278

KEY WORDS adherence; plagiocephaly; positional skull deformity; helmet; orthotic; health belief model; craniofacial

ABBREVIATIONS HBM $=$ health belief model; WHO $=$ World Health Organization .

SUBMITTED May 19, 2016. ACCEPTED September 12, 2016.

INCLUDE WHEN CITING Published online January 13, 2017; DOI: 10.3171/2016.9.PEDS16278. 
$\mathrm{T}$ HE institution of the Back to Sleep Campaign in 1992 has significantly decreased infant mortality due to sudden infant death syndrome (SIDS), while the prevalence of positional skull deformity has increased substantially. ${ }^{11,12}$ Positional plagiocephaly and brachycephaly are the most common deformities. Occipital plagiocephaly is characterized by an increased oblique diagonal difference with noticeable asymmetrical flattening of the occiput. Brachycephaly is characterized by anterior-posterior shortening and compensatory biparietal widening. ${ }^{6}$ Both plagiocephaly and brachycephaly are typically noted by $2-4$ months of age and have decreasing prevalence as children grow older. ${ }^{6}$ These deformities are frequently seen in association with torticollis. Treatment options for positional deformations include no intervention, repositioning, physical therapy, and use of a cranial orthosis (otherwise known as helmet therapy). ${ }^{13}$

If the degree of deformity warrants intervention, the first-line treatment is repositioning of the infant to overcome positional preference. Physical or occupational therapy is recommended when torticollis is the underlying cause of positional deformity. ${ }^{8}$ If these conservative measures are not effective, a custom cranial orthosis may be placed to aid in the correction of the deformity. ${ }^{13}$ The cranial orthosis takes advantage of the rapid rate of brain growth in normal infants and may modulate skull shaping by fitting closely to the head to prevent pressure on one area while still leaving room for preferential directed growth at the flattened area. These devices are typically worn for 23 hours per day for several weeks..$^{14,16}$

As with other therapeutic interventions, successful treatment of positional cranial deformities is likely to be related to the degree of compliance with prescribed treatment. Because improved compliance is generally thought to result in improved outcomes, factors affecting adherence to therapeutic recommendations have long been a subject of study. The WHO defines adherence as "the extent to which the patient follows medical instructions" and "the extent to which a person's behavior corresponds with recommendations from a health care provider."18 Early efforts to examine health care adherence led to the development of the health belief model (HBM), which identified psychological readiness, situational influences, and environmental conditions as the major factors contributing to adherence. ${ }^{5}$ Psychological readiness is influenced by perceived risk of contracting illness or severity of disease and belief that detection and treatment would be of benefit. Situational influences include personal factors, but also influence from others in favor of or against taking a specific action. Environmental influences include factors that alter accessibility to resources, affecting their utilization. ${ }^{5}$ The HBM was designed to predict factors that would drive behavior change, specifically, participation in a health screening program. ${ }^{5,7,10}$ Knowledge of these factors allows them to be taken into consideration when designing interventional programs to improve adherence to medical recommendations.?

More recently, a meta-analysis of relationships between adherence and outcomes conducted at the University of California at Riverside reported that adherence is highly correlated with positive treatment outcomes. Compared with non-adherence, adherence to treatment reduces the risk for a null treatment outcome by $26 \% .^{3}$ Similarly, a 2003 report from the World Health Organization (WHO), entitled "Adherence to Long-Term Therapies-Evidence for Action," further supported the idea that increasing patient adherence to recommended treatments will have a significant impact on treatment outcomes and overall health. ${ }^{18}$ Additionally, this report proposed a framework, similar to that of the HBM, which consisted of 5 components thought to affect adherence: "social- and economic-related" factors, health system factors, therapy-related factors, condition-related factors, and patient-related factors. $^{18}$

A number of social and economic factors have previously been identified as associated with improved adherence to various types of medical treatment. For example, type of insurance coverage was found to be associated with non-adherence to antihypertensive medication, ${ }^{1,2}$ mammography screening, ${ }^{4}$ and follow-up after orthopedic trauma surgery. ${ }^{17}$ The current study evaluated patients treated for positional cranial deformities at the Texas Children's Hospital Cranial Deformity Clinic to identify factors associated with parent-reported adherence to initial treatment recommendations and completion of long-term cranial remolding therapy. By identifying factors associated with poor compliance, we aimed to help patients and providers overcome these boundaries to improve treatment compliance and outcomes.

\section{Methods}

The Cranial Deformity Clinic was established in 2007 to reduce variation in management and improve coordination of care for infants with positional cranial deformities. A team of pediatric neurosurgeons and advanced practice providers screened all patients presenting to the Texas Children's Hospital Cranial Deformity Clinic between 2007 and 2014 for cranial deformation using a STARscanner laser surface scanner (Orthomerica Products Inc., Orlando, FL). Patients treated for positional cranial deformation (occipital plagiocephaly, occipital brachycephaly, or both) who were under the age of 12 months at presentation and who had not received treatment prior to being evaluated at Texas Children's Hospital were included in the study. Exclusion criteria included patients with ventriculoperitoneal shunts, severe medical/developmental issues, cephalohematomas, and normal head shapes. Patients with radiological diagnoses of craniosynostosis requiring surgical treatment and those who had received cranial orthosis treatment prior to presentation were also excluded. Adherence was recorded according to parental report on details of treatment at the time of each clinic visit. Our treatment algorithm and rationale are described in a related paper by Lam et al. ${ }^{8}$ This study received approval from the Baylor College of Medicine institutional review board.

\section{Model for Factors Affecting Adherence}

We used the 5-dimension model described in the 2003 WHO report ${ }^{18}$ to understand the factors associated with adherence to recommended treatment as the framework for this study. The likelihood of patients' adherence to 
recommended treatment is influenced by several factors, including individual perceptions and characteristics (demographics, socioeconomic status, peer reference) and perceived benefits/barriers to treatment. We examined factors in each of the 5 domains. Social and economic factors included high costs of treatment and access to the treatment facility-specifically, long distance from the treatment setting. Health systems factors in this study are fixed, as all patients in the study were treated in the same clinic by the same team of health care providers. Therapyrelated factors varied with the nature of treatment, which included repositioning, physical therapy, cranial orthosis therapy, or some combination thereof. Side effects were limited even in the group treated with cranial orthosis therapy, the most intensive therapeutic option. We did not directly measure condition-related factors, the fourth component of this framework, as it is difficult to assign a difference in disease severity to these patients beyond laser surface scan measurements. However, it is likely that the severity of the deformity contributes to the family's perception of severity and may affect treatment decisions. Finally, the patient-related factors that we examined were patient sex, race, and age..$^{5,10,18}$

\section{Statistical Analysis}

Multivariate logistic regression was applied to examine factors associated with parent-reported adherence to recommended treatment and model the factors associated with treatment completion. Risk factors with $p<0.2$ in univariate analysis were included in multivariate analysis. All statistical analyses were performed with SAS (SAS Institute). A p value of $<0.05$ was considered statistically significant.

\section{Results}

A total of 1299 patients were screened at the Texas Children's Hospital Head Shape Clinic between 2007 and 2014. (See Fig. 2 in the related article by Lam et al. ${ }^{8}$ ). We included patients younger than 12 months of age at presentation with a diagnosis of occipital brachycephaly, occipital plagiocephaly, or both, who had a documented recommended treatment plan. Of the 1299 patients screened, 991 met the criteria for inclusion in the study.

Characteristics of the included patients are described in Table 1. The average chronological age at presentation was 6.2 months; $76.3 \%$ of patients were between 4 and 9 months of age, and $69.3 \%$ were male. The majority of the children were white $(40.7 \%)$ or Hispanic (32.6\%). Slightly more than half of the patients $(51.7 \%)$ were covered by commercial insurance, while $38.7 \%$ had public insurance, defined as Medicaid/Children's Health Insurance Program (CHIP). The most commonly recommended initial treatment was repositioning or physical therapy, and $85.7 \%$ of parents reported adherence to the initially recommended treatment plan. Of 991 patients, 107 did not return for follow-up visits after the initial appointment. Lack of follow-up in these cases was considered loss to expected follow-up, rather than being classified as omission of optional follow-up.

Univariate analyses showed a difference in parent-re- ported adherence rates between several patient subgroups. Families with public insurance had an adherence rate of $80.2 \%$. Families with commercial insurance were more likely to adhere to the recommended treatment, with an adherence rate of $89.6 \%(\mathrm{p}=0.001)$. The nature of the diagnosis also affected adherence. Families of patients diagnosed with brachycephaly alone had an adherence rate of $79.9 \%$, compared with $86.4 \%$ and $90.8 \%$ for the diagnosis of plagiocephaly and the diagnosis of mixed plagiocephaly and brachycephaly, respectively $(p=0.017)$. Finally, the nature of recommended treatment affected adherence. Families of patients recommended for cranial orthosis therapy or cranial orthosis therapy in combination with repositioning and/or physical therapy had compliance rates of $75.6 \%$ and $72.3 \%$, respectively. These rates are significantly lower than the compliance rate of families of patients referred for physical therapy or repositioning alone or in combination, which was $94.8 \%(\mathrm{p}<0.001)$. None of the patient baseline features, including weight at presentation, corrected or chronological age at diagnosis, and distance from treatment facility, had a significant effect on family adherence with initial treatment recommendation (Table 2).

The multivariate logistic regression confirmed that factors associated with adherence to initially recommended treatment included primary payer, diagnoses, and the nature of the recommended treatment (Table 3). Families of patients who had commercial insurance were more likely to adhere to treatment than those with public insurance (OR 1.89, 95\% CI 1.19-2.99, $\mathrm{p}=0.007$ ). Additionally, patients with a diagnosis of brachycephaly alone were less likely to have caregiver adherence to initial treatment than those diagnosed with plagiocephaly (OR 1.84, 95\% CI $1.14-2.96, \mathrm{p}=0.011)$ or both plagiocephaly and brachycephaly (OR 3.25, 95\% CI 1.52-6.95, p = 0.002). Finally, families of patients initially recommended for cranial orthosis therapy or combination therapy that included a cranial orthosis were less likely to be adherent by parent report than those who were initially referred for repositioning and/or physical therapy alone (OR $0.16,95 \% \mathrm{CI}$ $0.1-0.26, \mathrm{p}<0.001$ and OR $0.14,95 \%$ CI $0.08-0.25$, p < 0.001 , respectively). There were no significant differences in adherence to initial treatment based on age at presentation or race.

Five hundred fifty-two patient families (55.7\%) completed the course of recommended treatment with a final follow-up appointment at the end of therapy. Factors associated with treatment completion included corrected age at presentation, insurance type, diagnosis, nature of the recommended treatment, and distance between the patients' homes and the provider's office (Table 4). Families of patients who were younger at presentation $(0-4$ months) or who had commercial insurance (OR 1.49, $95 \%$ CI $1.10-2.02, \mathrm{p}=0.009$ ), a diagnosis of both brachycephaly and plagiocephaly (OR 2.26, 95\% CI 1.31-3.90, $\mathrm{p}=$ 0.003 ), recommendation for cranial orthosis therapy (OR $4.55,95 \%$ CI 3.24-6.38, $\mathrm{p}<0.001$ ), or recommendation for combined cranial orthosis, repositioning, and physical therapy (OR 3.19, 95\% CI 2.07-4.09, p < 0.001), and those who resided within 12 miles of the provider facility (OR 1.40, 95\% CI 1.00-1.96, $\mathrm{p}=0.047$ ) were more like- 
TABLE 1. Baseline patient characteristics by adherence to recommended treatment

\begin{tabular}{|c|c|c|c|c|c|c|c|}
\hline \multirow[b]{2}{*}{ Variable } & \multicolumn{2}{|c|}{ All Patients } & \multicolumn{2}{|c|}{$\begin{array}{c}\text { Adhered to } \\
\text { Recommended Treatment }\end{array}$} & \multicolumn{2}{|c|}{$\begin{array}{c}\text { Did Not Adhere to } \\
\text { Recommended Treatment }\end{array}$} & \multirow{2}{*}{$\begin{array}{c}p \\
\text { Value* }^{*}\end{array}$} \\
\hline & $\mathrm{N}$ & $\%$ & $\mathrm{~N}$ & $\%$ & $\mathrm{~N}$ & $\%$ & \\
\hline Total & 991 & & 849 & 85.7 & 142 & 14.3 & \\
\hline $\begin{array}{l}\text { Corrected age at presentation } \\
\text { in mos } \dagger\end{array}$ & & & & & & & $0.214 \ddagger$ \\
\hline $0-4$ & 193 & 19.5 & 173 & 89.6 & 20 & 10.4 & \\
\hline$>4-6$ & 428 & 43.2 & 363 & 84.8 & 65 & 15.2 & \\
\hline$>6-11$ & 370 & 37.3 & 313 & 84.6 & 57 & 15.4 & \\
\hline Sex & & & & & & & 0.615 \\
\hline Female & 304 & 30.7 & 263 & 86.5 & 41 & 13.5 & \\
\hline Male & 687 & 69.3 & 586 & 85.3 & 101 & 14.7 & \\
\hline Race & & & & & & & $0.060 \ddagger$ \\
\hline White & 403 & 40.7 & 361 & 89.6 & 42 & 10.4 & \\
\hline Black & 41 & 4.1 & 35 & 85.4 & 6 & 14.6 & \\
\hline Hispanic & 323 & 32.6 & 265 & 82.0 & 58 & 18.0 & \\
\hline Asian/other & 67 & 6.8 & 56 & 83.6 & 11 & 16.4 & \\
\hline Unknown & 157 & 15.8 & 132 & 84.1 & 25 & 15.9 & \\
\hline Insurance & & & & & & & $0.001 \ddagger$ \\
\hline Medicaid/CHIP & 384 & 38.7 & 308 & 80.2 & 76 & 19.8 & \\
\hline Commercial & 512 & 51.7 & 459 & 89.7 & 53 & 10.4 & \\
\hline Others & 81 & 8.2 & 70 & 86.4 & 11 & 13.6 & \\
\hline Unknown & 14 & 1.4 & 12 & 85.7 & 2 & 14.3 & \\
\hline Diagnosis & & & & & & & $0.017 \ddagger$ \\
\hline Brachycephaly & 182 & 18.4 & 145 & 79.7 & 37 & 20.3 & \\
\hline Plagiocephaly & 689 & 69.5 & 595 & 86.4 & 94 & 13.6 & \\
\hline Both & 120 & 12.1 & 109 & 90.8 & 11 & 9.2 & \\
\hline Recommended treatment & & & & & & & $<0.001 \ddagger$ \\
\hline $\mathrm{CO}$ & 311 & 31.4 & 235 & 75.6 & 76 & 24.4 & \\
\hline $\mathrm{RP} / \mathrm{PT}$ & 543 & 54.8 & 515 & 94.8 & 28 & 5.2 & \\
\hline $\mathrm{RP} / \mathrm{PT} / \mathrm{CO}$ & 137 & 13.8 & 99 & 72.3 & 38 & 27.7 & \\
\hline \multicolumn{8}{|l|}{ Distance to provider in miles } \\
\hline$<12(\mathrm{Q} 1)$ & 247 & 25.1 & 216 & 84.7 & 31 & 12.6 & 0.379 \\
\hline$\geq 12$ & 736 & 74.9 & 627 & 86.1 & 109 & 14.8 & \\
\hline
\end{tabular}

ly to complete treatment when compared with families of patients older than 4 months at presentation (age 4-6 months, OR 0.046, 95\% CI 0.31-0.69, p < 0.001; age 7-11 months, OR $0.015,95 \%$ CI $0.10-0.22, p<0.001$ ), with a diagnosis of brachycephaly only, recommended treatment of repositioning with or without physical therapy, or residing more than 12 miles from the provider's office. Twelve miles was chosen as the cut-off for analysis as it was the first quartile distance of travel for all patients in the cohort.

\section{Discussion}

The current study examined factors affecting parentreported adherence to initial treatment recommendations and completion of recommended therapy among families of patients diagnosed with positional cranial deformities at Texas Children's Hospital between 2007 and 2014. We aimed to identify patient and treatment factors that predict adherence with initial recommendations and longterm therapy in these patient families. These factors have been classified according to the framework published by the $\mathrm{WHO},{ }^{18}$ which identifies social and economic factors, health system factors, therapy-related factors, conditionrelated factors, and patient-related factors as the principle components contributing to or detracting from adherence to recommended treatment. Awareness of these factors can help health care professionals approach treatment of these patients and their families in ways that will maxi- 
TABLE 2. Baseline patient characteristics and distance to provider stratified by adherence to recommended treatment

\begin{tabular}{|c|c|c|c|c|c|}
\hline $\begin{array}{c}\text { Characteristic } \\
\text { \& Treatment } \\
\text { Adherence }\end{array}$ & $\begin{array}{c}\text { No. } \\
\text { of } \\
\text { Pts }\end{array}$ & Mean (SD) & $95 \% \mathrm{Cl}$ & Min, Max & $\begin{array}{c}p \\
\text { Value }\end{array}$ \\
\hline \multicolumn{6}{|l|}{$\begin{array}{l}\text { Weight at diagno- } \\
\text { sis in Ibs }\end{array}$} \\
\hline All & 860 & $7.8(1.7)$ & $7.7-7.9$ & $1.1,11.3$ & 0.671 \\
\hline Adherent & 736 & $7.8(1.7)$ & $7.7-7.9$ & $1.1,11.3$ & \\
\hline Not adherent & 124 & $7.9(1.4)$ & $7.6-8.1$ & $4,11.1$ & \\
\hline \multicolumn{6}{|l|}{$\begin{array}{l}\text { Corrected age in } \\
\text { mos* }^{*}\end{array}$} \\
\hline All & 991 & $5.8(1.9)$ & $5.6-5.9$ & $0.7,11$ & 0.397 \\
\hline Adherent & 849 & $5.7(1.9)$ & $5.6-5.9$ & $0.7,11$ & \\
\hline Not adherent & 142 & $5.9(1.7)$ & $5.6-6.2$ & 0.7 .11 & \\
\hline \multicolumn{6}{|l|}{$\begin{array}{l}\text { Chronological age } \\
\text { in } \text { mos }^{*}\end{array}$} \\
\hline All & 991 & $6.2(1.8)$ & $6.1-6.3$ & 1,11 & 0.479 \\
\hline Adherent & 849 & $6.2(1.9)$ & $6.1-6.3$ & 1,11 & \\
\hline Not adherent & 142 & $6.3(1.5)$ & $6.1-6.6$ & 3,11 & \\
\hline \multicolumn{6}{|l|}{$\begin{array}{l}\text { Distance to pro- } \\
\text { vider in miles }\end{array}$} \\
\hline All & 983 & $31.5(81.3)$ & $20.6-37.2$ & $0,1613.1$ & 0.684 \\
\hline Adherent & 843 & $31.9(85.4)$ & $26.1-37.7$ & $0,1613.1$ & \\
\hline Not adherent & 140 & $28.9(49.5)$ & $26.4-36.6$ & $1.4,516.0$ & \\
\hline
\end{tabular}

mize the opportunity for adherence. In practice, we now take socioeconomic factors into account when developing treatment and planning follow-up.

Encouraging adherence to treatment is a key component of completing future high-quality studies to assess the clinical utility of cranial orthosis use and its benefit in correcting positional plagiocephaly in young children. Identifying and addressing barriers to adherence is an important component of this encouragement. Furthermore, a meta-analysis reviewing the relationship between patient adherence and treatment outcomes suggested that the correlation between adherence and outcomes is more tightly intertwined in pediatric patients than in adults. ${ }^{3}$ These findings can likely be extrapolated to include treatment for correction of positional plagiocephaly. A significant step in both determining the relationship between treatment adherence and outcomes and improving patient adherence to treatment in patients being managed for positional deformities is identification of factors associated with good and poor treatment compliance.

The current study suggests that families of infants with positional deformities are less likely to adhere to recommended treatment when that treatment is cranial orthosis therapy than when it is repositioning or physical therapy (Table 3). However, both younger age and use of cranial orthosis were associated with increased likelihood of completing treatment (Table 4). This suggests that use of a cranial orthosis represents a barrier to treatment initiation but that parents who commit to cranial orthosis therapy
TABLE 3. Factors associated with adherence to initial recommended treatment

\begin{tabular}{|c|c|c|c|}
\hline Factor & OR & $95 \% \mathrm{Cl}$ & $p$ Value \\
\hline \multicolumn{4}{|c|}{$\begin{array}{l}\text { Corrected age at presentation } \\
\text { in mos }\end{array}$} \\
\hline $0-4$ & $\operatorname{Ref}=1$ & & \\
\hline$>4-6$ & 1.00 & $0.56-1.79$ & 0.991 \\
\hline$>6-9$ & 0.87 & $0.48-1.57$ & 0.635 \\
\hline$>9$ & 1.22 & $0.32-4.66$ & 0.776 \\
\hline \multicolumn{4}{|l|}{ Race } \\
\hline White & $\operatorname{Ref}=1$ & & \\
\hline Black & 1.00 & $0.37-2.68$ & 0.996 \\
\hline Hispanic & 0.74 & $0.44-1.23$ & 0.245 \\
\hline Asian/other & 0.59 & $0.27-1.29$ & 0.185 \\
\hline Unknown & 0.70 & $0.39-1.25$ & 0.231 \\
\hline \multicolumn{4}{|l|}{ Insurance } \\
\hline Medicaid/CHIP & $\operatorname{Ref}=1$ & & \\
\hline Commercial & 1.89 & $1.19-2.99$ & $0.007^{*}$ \\
\hline Others & 1.50 & $0.72-3.11$ & 0.278 \\
\hline Unknown & 1.87 & $0.38-9.60$ & 0.449 \\
\hline \multicolumn{4}{|l|}{ Diagnosis } \\
\hline Brachycephaly & $\operatorname{Ref}=1$ & & \\
\hline Plagiocephaly & 1.84 & $1.14-2.96$ & $0.011^{*}$ \\
\hline Both & 3.25 & $1.52-6.95$ & $0.002^{*}$ \\
\hline \multicolumn{4}{|c|}{ Recommended treatment } \\
\hline RP/PT & $\operatorname{Ref}=1$ & & \\
\hline $\mathrm{CO}$ & 0.16 & $0.10-0.26$ & $<0.001^{*}$ \\
\hline RP/PT/CO & 0.14 & $0.08-0.25$ & $<0.001^{*}$ \\
\hline
\end{tabular}

Ref $=$ reference.

* Interpretation: $O R<1$, less chance to follow medical advice; $O R>1$, greater chance to follow medical advice.

are subsequently more likely to follow through with the entire course of treatment. This finding is not surprising when considered in the context of the WHO framework for adherence. Cranial orthosis therapy is more cumbersome with regard to expense, acquisition of equipment, and effort on the part of parent and child. This therapeutic option thus imposes an economic burden on patient families and is limited by patient-related factors, such as understanding the treatment plan and motivation to ensure compliance if family members perceive that the infant is uncomfortable. Additionally, social factors, such as parental concern about the social stigma surrounding cranial orthosis therapy and uncertainty of the effectiveness of treatment, ${ }^{15}$ may discourage initial compliance.

Therapy-related and economic factors are also likely to contribute to lack of adherence in regimens including cranial orthosis therapy. Medicaid and some other providers may not cover orthotic cranial orthoses for the treatment of positional deformity in children. This treatment option may, therefore, be significantly more costly for certain patients, while repositioning the child has no associated expense. This factor may partly explain why patients with public insurance showed lower compliance with both initial treatment and completion of therapy. Finally, poor 
TABLE 4. Factors associated with completion of entire treatment course

\begin{tabular}{|c|c|c|c|}
\hline Factor & OR & $95 \% \mathrm{Cl}$ & p Value \\
\hline \multicolumn{4}{|c|}{ Corrected age at presentation in mos } \\
\hline $0-4$ & $\operatorname{Ref}=1$ & & \\
\hline$>4-6$ & 0.46 & $0.31-0.69$ & $<0.001^{*}$ \\
\hline$>6-11$ & 0.15 & $0.10-0.22$ & $<0.001^{*}$ \\
\hline \multicolumn{4}{|l|}{ Sex } \\
\hline Female & $\operatorname{Ref}=1$ & & \\
\hline Male & 1.13 & $0.83-1.53$ & 0.436 \\
\hline \multicolumn{4}{|l|}{ Insurance } \\
\hline Medicaid/CHIP & $\operatorname{Ref}=1$ & & \\
\hline Commercial & 1.49 & $1.10-2.02$ & $0.009^{*}$ \\
\hline Others & 1.31 & $0.76-2.26$ & 0.326 \\
\hline Unknown & 4.49 & $0.53-37.94$ & 0.168 \\
\hline \multicolumn{4}{|l|}{ Diagnosis } \\
\hline Brachycephaly & $\operatorname{Ref}=1$ & & \\
\hline Plagiocephaly & 1.25 & $0.86-1.82$ & 0.246 \\
\hline Both & 2.26 & $1.31-3.90$ & $0.003^{*}$ \\
\hline \multicolumn{4}{|c|}{ Recommended treatment } \\
\hline $\mathrm{RP} / \mathrm{PT}$ & $\operatorname{Ref}=1$ & & \\
\hline $\mathrm{CO}$ & 4.55 & $3.24-6.38$ & $<0.001^{*}$ \\
\hline $\mathrm{RP} / \mathrm{PT} / \mathrm{CO}$ & 3.19 & $2.07-4.90$ & $<0.001^{*}$ \\
\hline \multicolumn{4}{|c|}{ Distance to provider in miles } \\
\hline$>12$ & $\operatorname{Ref}=1$ & & \\
\hline$\leq 12$ (Q1) & 1.40 & $1.00-1.96$ & $0.047^{*}$ \\
\hline
\end{tabular}

tolerance of therapy, including skin breakdown and resistance of the child to treatment, is more likely to occur in the group treated with cranial orthoses, which results in a negative effect on adherence.

Diagnosis was the third factor identified as having an influence on adherence to initial treatment recommendation and completion of therapy. Diagnosis of plagiocephaly and dual diagnosis of both brachycephaly and plagiocephaly were associated with increased initial treatment compliance, while only dual diagnosis was associated with increased likelihood of treatment completion. This finding was also unsurprising, as diagnosis of plagiocephaly and diagnosis of concurrent plagiocephaly and brachycephaly are more likely to be perceived by families as true deformity, while brachycephaly, being more symmetrical, is not. A greater degree of apparent deformity is a conditionrelated factor that heavily favors therapy. Additionally, in these cases social pressures may encourage treatment compliance, as the deformity is perceived to be more noticeable to others.

Young age at presentation, defined as 0-4 months, did not predict adherence to initial recommendations. However, it was associated with increased likelihood of eventual treatment completion. This suggests that parents of patients who present at younger ages are better able to comply with treatment, due to one or more of the WHO report framework components. For example, presentation at a younger age may indicate a more severe deformity, a perceived condition factor, which could enhance parental motivation to adhere to treatment. Environmental conditions may also be more favorable, as younger children may be less able to resist treatment efforts, which makes compliance easier for parents. Finally, treatment factors, in this case the nature of treatment, may favor compliance in younger children. Our treatment paradigm generally recommends repositioning/physical therapy, which we have shown to have a higher rate of compliance than cranial orthosis therapy for younger children.

Finally, patients who resided within 12 miles of Texas Children's Hospital were more likely to complete treatment than those residing more than 12 miles from the provider's office. Travel distance is a clear patient and economic factor that may reduce compliance. Increased travel time increases cost and time off from work. Additionally, increased travel time requires a greater investment in treatment and higher levels of motivation to maintain adherence, thus making completion of therapy less likely.

\section{Strengths of the Current Study}

The current study reflects a large study population in which each patient was offered similar evaluation and follow-up. Each patient was seen and counseled by a pediatric neurosurgeon in the head shape screening team. In addition to clinical evaluation and judgment about the degree of deformity, the surface laser STAR scanner was used to track objective measurements, providing a reliable measure of treatment effect. Additionally, because many of these patients come to Texas Children's Hospital for comprehensive care, we had access to a great deal of demographic information.

\section{Limitations of the Current Study}

A significant limitation of the current study is the dependence on self-reported behavior. We were unable to verify how long patients wore the cranial orthosis at home or how frequently or diligently they pursued repositioning and physical therapy. Our only direct measure for compliance was continued appearance at follow-up appointments. Additionally, this is a retrospective study and, as such, the study relies on chart review. The clinical data are thus incomplete. Patients were not randomized to treatment groups; therefore, patient-specific factors and parental preferences may have influenced the treatment decisions at the time, introducing bias. The study is also observational in nature and reflects the treatment algorithm developed in our practice. Thus there is no comparison group. For this reason, we cannot compare the effects of adherence with initial treatment on outcome. Assessment of the efficacy of treatment was further limited by lack of encouragement for follow-up in conservatively managed patients with mild deformity. This also resulted in a large number of patients with incomplete follow-up, unfavorably skewing completion data. Finally, there are important factors, such as income, family stressors, educational level, and attitudes toward health care, that are considered as possible factors affecting compliance according to the HBM and WHO framework in the current study but have not been evaluated on an individual basis here. There are 
many factors that can contribute to caregiver's perception of the severity of the problem. One could posit that other factors may include race, ethnicity, birth order, number of children in the home, family history and family medical experiences, identity of primary caregiver, socioeconomic status, geography, peer groups, and parent occupation. We are not able to provide a comprehensive assessment of this problem in a retrospective study; however, these would all be considerations in future prospective studies.

This is a single-center retrospective study, and as such, it is not known how much of the findings can be generalized to other centers, other regions, and other patient populations. The influence of geography and transportation, locations and accessibility of orthotics and clinic offices, Medicaid and insurance coverage, and the ethnicity and socioeconomic make-up of the population all may be specific to a center. However, understanding the influence of nonclinical factors is an increasingly important part in the practice of medicine.

\section{Conclusions}

As demonstrated with numerous other treatment modalities, adherence to a recommended treatment regimen is likely associated with improved outcomes in treatment of positional cranial deformities in infants. Identification of factors associated with poor adherence to initial treatment or decreased rate of completion of treatment can aid in the development of treatment strategies and support for patients and families to improve adherence. The current study indicates that less complex diagnosis, costly treatment, and public health insurance are associated with lower rates of parent-reported adherence to treatment, while more complex diagnosis, private insurance, and more conservative treatment were associated with better initial adherence. In addition, once patients initiated the treatment, higher likelihood of completing full treatment course was associated with younger age, commercial insurance, more complex diagnosis, cranial orthosis treatment, and shorter distance from the treatment center to home. In clinical practice, these socioeconomic factors can be taken into account when developing treatment and follow-up planning.

\section{References}

1. AlGhurair SA, Hughes CA, Simpson SH, Guirguis LM: A systematic review of patient self-reported barriers of adherence to antihypertensive medications using the world health organization multidimensional adherence model. J Clin Hypertens (Greenwich) 14:877-886, 2012

2. Bernard DM, Johansson P, Fang Z: Out-of-pocket healthcare expenditure burdens among nonelderly adults with hypertension. Am J Manag Care 20:406-413, 2014

3. DiMatteo MR, Giordani PJ, Lepper HS, Croghan TW: Patient adherence and medical treatment outcomes: a meta-analysis. Med Care 40:794-811, 2002

4. Henry KA, McDonald K, Sherman R, Kinney AY, Stroup AM: Association between individual and geographic factors and nonadherence to mammography screening guidelines. J Womens Health (Larchmt) 23:664-674, 2014

5. Hochbaum GM: Public Participation in Medical Screening Programs: A Socio-Psychological Study. Washington, DC: US Public Health Service, 1958

6. Hutchison BL, Hutchison LA, Thompson JM, Mitchell EA:
Plagiocephaly and brachycephaly in the first two years of life: a prospective cohort study. Pediatrics 114:970-980, 2004

7. Jones CJ, Smith H, Llewellyn C: Evaluating the effectiveness of health belief model interventions in improving adherence: a systematic review. Health Psychol Rev 8:253-269, 2014

8. Lam S, Pan IW, Strickland BA, Hadley C, Daniels B, Brookshier J, et al: Factors influencing outcomes of the treatment of positional plagiocephaly in infants: a 7-year experience. J Neurosurg Pediatr [epub ahead of print January 13, 2017. DOI: $10.31717 / 2016.9 . P E D S 16275]$

9. Laughlin J, Luerssen TG, Dias MS: Prevention and management of positional skull deformities in infants. Pediatrics 128:1236-1241, 2011

10. Rosenstock IM, Strecher VJ, Becker MH: Social learning theory and the Health Belief Model. Health Educ Q 15:175183, 1988

11. Sheu SU, Ethen MK, Scheuerle AE, Langlois PH: Investigation into an increase in plagiocephaly in Texas from 1999 to 2007. Arch Pediatr Adolesc Med 165:708-713, 2011

12. Shweikeh F, Nuño M, Danielpour M, Krieger MD, Drazin D: Positional plagiocephaly: an analysis of the literature on the effectiveness of current guidelines. Neurosurg Focus 35(4):E1, 2013

13. Teichgraeber JF, Seymour-Dempsey K, Baumgartner JE, Xia JJ, Waller AL, Gateno J: Molding helmet therapy in the treatment of brachycephaly and plagiocephaly. J Craniofac Surg 15:118-123, 2004

14. van Vlimmeren LA, Helders PJ, van Adrichem LN, Engelbert $\mathrm{RH}$ : Torticollis and plagiocephaly in infancy: therapeutic strategies. Pediatr Rehabil 9:40-46, 2006

15. van Wijk RM, van Til JA, Groothuis-Oudshoorn CG, L'Hoir MP, Boere-Boonekamp MM, IJzerman MJ: Parents' decision for helmet therapy in infants with skull deformation. Childs Nerv Syst 30:1225-1232, 2014

16. van Wijk RM, van Vlimmeren LA, Groothuis-Oudshoorn CG, Van der Ploeg CP, IJzerman MJ, Boere-Boonekamp MM: Helmet therapy in infants with positional skull deformation: randomised controlled trial. BMJ 348:g2741, 2014

17. Whiting PS, Greenberg SE, Thakore RV, Alamanda VK, Ehrenfeld JM, Obremskey WT, et al: What factors influence follow-up in orthopedic trauma surgery? Arch Orthop Trauma Surg 135:321-327, 2015

18. World Health Organization: Adherence to Long-Term Therapies: Evidence for Action. Geneva: World Health Organization, 2003

\section{Disclosures}

The authors report no conflict of interest concerning the materials or methods used in this study or the findings specified in this paper.

\section{Author Contributions}

Conception and design: Pan, Lam. Acquisition of data: Luerssen, Hadley, Daniels, Strickland, Brookshier. Analysis and interpretation of data: Pan. Drafting the article: Pan, Lam, Hadley. Critically revising the article: Pan, Lam, Luerssen, Daniels, Strickland. Reviewed submitted version of manuscript: Pan, Lam, Luerssen, Brookshier. Statistical analysis: Pan. Administrative/technical/ material support: Luerssen, Brookshier. Study supervision: Lam, Luerssen.

\section{Correspondence}

I-Wen Pan, Department of Neurosurgery, Baylor College of Medicine, Texas Children's Hospital, 6701 Fannin St., Ste. 1230, Houston, TX 77030. email: iepan@texaschildrens.org. 